\title{
Aspectos do problema das cáries dentárias no Estado do Amazonas
}

\author{
Roger Shrimpton ( ${ }^{*}$ ) \\ Rodolfo Giugliano ("*)
}

\section{Resumo}

São apresentados dados sobre a incidência de cáries em 193 crianças pobres de 1 a 8 anos de idade em Manaus, Amazonas, 1976. A incidência de cáries foi de $77,7 \%$, sendo que no grupo etário de 8 anos atingiu a $97,9 \%$. Possíveis fatores locais envolvicios na evolução das cáries e as possíveis medidas a serem tomadas são discutidos. São enfatizadas a inapropriedade do tratamento dentário conservador e a essencialidade de medidas preventivas nesta área tão grande mas esparsamente povoada. A promoção da amamentação sobre a alimentação artificial nas primeiras semanas de vida da criança e a fluoretação do sal, são sugeridas como medidas práticas de mínimo custo e máximo alcance na redução das cáries em populações rurais e urbanas da Bacia Amazônica.

\section{INTRODUÇAO}

A cárie dentária é uma das doenças infecciosas predominantes do homem (Gibbons \& Van Houte, 1975).

Um levantamento nutricional na Bacia Amazônica, nos anos de 1954 a 1956, demonstrou a incidência de $56,1 \%$ de cáries dentárias em 4.293 pessoas examinadas (Silva, 1959). O levantamento demonstrou que cáries já eram bastante comuns em crianças de pouca idade (Lowenstein, 1967), mas na realidade $56 \%$ é pouco, considerando que mais de $80 \%$ das crianças de países desenvolvidos já sofrem de cáries com 10 anos de idade (Wei, 1975). De 1950 para cá, as zonas urbanas amazônicas sofreram grandes aumentos, passando de $25 \%$ para $53 \%$ da populaçâo total (Anuário Estatístico do Amazoriás, 1975) .

O objetivo deste trabalho é apresentar dados preliminares sobre cáries dentárias em Manaus e enfatizar os aspectos preventivos importantes ao controle dessa doença num Estado, vasto como o Amazonas.

\section{MAteriais e MÉtodos}

Os resultados apreseritados nesta publicação foram coletadios em 2 levantamentos em crianças de Manaus, entre 132 crianças em idade pré-escolar (Giugliano \& Shrimpton, 1977a) e 71 crianças no $1 .^{\circ}$ ano escolar de uma escola em área pobre de Manaus (Giugliano \& Shrimpton, s/d.). O número de dentes muito comprometidos, obturados ou faltantes foram observados e registrados por um pediatra, não um dentista, baseados nos critérios e normas seguidos no Inquérito Nutricional (ICNND, 1963).

\section{Resultados}

Os resultados dos dois grupos de crianças foram reunidos e apresentados na Tabela I. Os dentes ausentes não são apresentados, pois, nesta idade muitas crianças estão na fase de troca de dentição de leite para os dentes permanentes. A população estudada mostra que

\section{TABELA I}

Incidência de cáries dentárias numa população de crianças pobres de 1 a 8 anos de idade em Manaus, 1976

\begin{tabular}{|c|c|c|c|c|c|c|}
\hline \multirow[b]{2}{*}{$\begin{array}{l}\text { Idade } \\
\text { em } \\
\text { anos }\end{array}$} & \multirow{2}{*}{$\begin{array}{l}\mathrm{N} . \mathrm{O}^{\circ} \text { de } \\
\text { crianças } \\
\text { exami- } \\
\text { nadas }\end{array}$} & \multicolumn{2}{|c|}{ INCIDENCIA DE } & \multirow{2}{*}{$\frac{\text { CARIES * }}{5+}$} & \multirow[b]{2}{*}{$\begin{array}{l}\text { Total de } \\
\text { crianças } \\
\text { c/ cáries }\end{array}$} & \multirow[b]{2}{*}{$\begin{array}{l}\% \text { crian- } \\
\text { sos cim } \\
\text { cáries }\end{array}$} \\
\hline & & $1-2$ & $3-4$ & & & \\
\hline 1 & 3 & 0 & 0 & 0 & 0 & 0 \\
\hline 2 & 17 & 3 & 1 & 0 & 4 & 23,5 \\
\hline 3 & 14 & 3 & 1 & 1 & 5 & 35,7 \\
\hline 4 & 24 & 5 & 6 & 3 & 14 & 58,3 \\
\hline 5 & 24 & 5 & 8 & 5 & 18 & 75,0 \\
\hline 6 & 21 & 8 & 4 & 7 & 19 & 90,5 \\
\hline 7 & 36 & 9 & 11 & 13 & 33 & 91,7 \\
\hline 8 & 48 & 9 & 17 & 23 & 47 & 97,9 \\
\hline Total & 193 & & & & 150 & 77,7 \\
\hline
\end{tabular}

(*) - Instituto Nacional de Pesquisas da Amazônia, Manaus.

(**) - Instituto Nacional de Pesquisas da Amazônia e Hospital de Moléstias Tropicais, Manaus. 
com a idade, a porcentagem de crianças com dentes atacados aumenta, e aos 8 anos, $97,9 \%$ já estão afetadas.

\section{DISCUSSÃo}

Embora seja uma pequena amostra, os resultados sugerem que a situação das cáries dentárias dos habitantes do Amazonas apresentada pelo Inquérito Nutricional do Conselho $\mathrm{Na}$ cional de Alimentação (Silva, 1959) não mais é válida na zona urbana de Manaus. Isso não é surpreendente e concorre com as incidências encontradas no resto do Brasil (Grinplastch, 1974)

Não existem estudos de cáries dentárias na zona rural do Amazonas. Hanson (1933) anotou que o indio geralmente tem bons dentes, mas uma vez em contato com nossa civilização, as cáries logo aparecem. Seria interessante estudar a flora bucal dos índios não civilizados, para verificar se os fatores envolvidos na evolução das cáries em índios aculturadios são a dieta ou a infecção com bactérias responsáveis pelas cáries (Michalek et al. 1976). Eniretanto, não säo todos os índios que lêm bons dentes (Velez Boza \& Baumgartner, 1962) .

A Bacia Amazônica, como área de pesquisa, oferece amplo campo de trabalho para resolver os mitos sobre a relação entre dieta e evolução das cáries.

Cáries resultam da interação dos dentes, bactérias e resíduos alimentares na cavidade bucal. Fatores envolvidos na preponderância das cáries incluem a composição química do esmalte, hábitos de higiene, a presença de bactérias cariogênicas e a presença de substratos facilmente fermentáveis nos alimentos consumidos (Wilson et al., 1975) .

O fato de que apenas $12 \%$ das mães de crianças, em estudo anterior no bairro de Coroado, tenham procurado o dentista para fazer obturações, enquanto $13 \%$ nunca foram ao dentista, indica o estado precário do atendimento dentário a esta população. Sessenta e três porcento das mães foram ao dentista, mas apenas para fazer extraçōes dentárias e $37 \%$ tinham prótešes (dados não publicados). Das crianças examinadas nos inquéritos, somente $36 \%$ (7) tinham obturações, enquanto
$77,7 \%$ (150) tinham cáries. O tratamento dentário conservador é caro e não representa uma possível solução desse problema, para a maioria da população, enquanto o INPS provê apenas pela extração dos dentes. A prevenção é a melhor e única alternativa.

A esperança de se diminuir a incidência de cáries através da promoção de melhor higiene bucal nessa população nâo é realista. O consumo de sucrose em populações urbanas na Amazônia é praticamente generalizada (Silva, 1959; Lira, 1960; Shrimpton \& Rodrigues, s/d). devido ao largo consumo de café forte e doce. As probabilidades, de tais hábitos alimentares serem modificados, săo desprezíveis. A promoção do açúcar não refinado, que é um agente menos cariogênico do que o açúcar refinado, poderia ser afetada por manipulações de preço e subsídios.

O esmalte do dente é diferente de qualquer outro tecido calcificado do corpo, pois não possui conexōes capilares ou linfáticas. Quando o dente surge na cavidade bucal, cessa o suprimento sanguíneo do esmalte. Os dentes não têm mecanismo de reabsorção e são capazes de proteger a si mesmos contra deficiências nutricionais melhor do que o esqueleto. O único efeito provocável no esmalte dentário é a redução da sua formação - ou hipoplasia do esmalte (Irving, 1957; Gibbons \& Van Houte, 1975) .

A composição química do dente é grandemente decidida nos primeiros anos de vida. A época do nascimento, todos os 20 dentes decíduos estão em um avançado estágio de calcificaçäc e desenvolvimento, e os 4 molares que aparecem aos 6 anos de idade começam a calcificar nos ossos maxilares (Wilson et al., 1975) .

As necessidades de cálcio do feto durante a gravidez correspondem a somente $2,5 \%$ do total de cálcio existente no corpo materno (Hytten \& Leich, 1964) e assim fica garantido o suprimento de cálcio para a formação do esmalte do feto, independente da dieta alimentar.

Se uma criança for amamentada durante os seis primeiros meses de vida, os requisitos minerais para o ótimo desenvolvimento do es- 
malte dentário estarão garantidos. A composição do leite humano é extraordinariamente constante, indiferente do consumo materno de alimentos (Thompson \& Black, 1975). A introdução do leite artificial nas primeiras duas semanas de vida provoca uma hipocalcemia neonatal. Apesar do leite de vaca conter mais cálcio que o leite humano, possui também muito mais fosfato. Neste estágio do desenvolvimento, os rins do recém-nascido não são capazes de utilizar esta dieta, em proporções não balanceadas de cálcio e fosforo, o que resulta em uma hipocalcemia que produz hipoplasia do esmalte, e, em conseqüência, dentes menos resistentes (Jaffe et al., 1973). A introdução da alimentação artificial nas duas primeiras semanas de vida, que foi por nós observado e é prática habitual em todas as maternidades em Manaus, deve ser fortemente combatida.

Quando a erupção dentária se inicia, o esmalte é relativamente imaturo e uma considerável mineralização toma lugar imediatamente após, modificando a rigidez da superfície dentária e a densidade mineral. Durante o processo de mineralização, a saliva é o agente mais importante no processo pós-eruptivo (Wei, 1974) .

Dos fatores dietéticos com efeito tópico sobre o esmalte e com importância no desenvolvimento das cáries, a sucrose foi mencionada como sendo o mais potente agente cariogênico. Também foi demonstrado que o fosfato na dieta alimentar é um efetivo agente cariostático (Scherp, 1971). A dieta local é possivelmente baixa em fósforo. O consumo de leite e queijo é baixo (Silva, 1959; Lira, 1960; Shrimpton \& Rodrigues, s/d). Os cereais consumidos em Manaus são altamente refinados, sendo que na farinha de trigo local a extração se aproxima de $80 \%$. O fitato no farelo de cereal é inibidor de cáries, provavelmente por causa do alto teor de fosfato, (McClure, 1960). Além disso, já foi demonstrado que o pão branco (pão de sal comum) tem uma capacidade cariogênica tão grande quanto a sucrose (Lambrou, 1974). Uma opção aberta ao estudo seria permitir taxas de extração de trigo de $90 \%$, para melhorar assim a qualidade nutritiva do pão.
Foi encontrada deficiência de fósforo em pastagens de gados em solos de terra firme do Vale do Amazonas (Sutmoeler et al., 1966). A adequação do consumo de fósforo nas dietas amazônicas requer estudos de avaliação.

No inquérito nutricional na Bacia Amazônica (Silva, 1956), na década de 50 , foi notado o baixo consumo de cálcio, mas não foram registrados sinais de deficiência de cálcio. O que não é surpreendente, pois a síndrome de deficiência de cálcio nunca foi demonstrada. em populações humanas em parte alguma do mundo, mesmo quando dietas possuiam um baixo teor de cálcio (Irwin \& Reinholz, 1973; ICNND, 1963). Pesquisas recentes sugerem, entretanto, que a moléstia periodontal espontânea, que é a rareficação do cemento e ossos dos maxilares que suportam os dentes causando a perda dos mesmos, possa responder positivamente à suplementação de cálcio (Krook et al., 1972).

Concentrações extremamente baixas de cálcio foram encontradas nas águas, sedimentos e peixes de regiões de águas pretas (Geisler \& Schneider, 1976). Deve ser lembrado, contudo, que as cadeias alimentares das quais o Homem depende, são geralmente longas e os elementos minerais se concentram no percurso. Foram encontrados teores normais de cálcio em folhas de árvores que cresciam em áreas inundadas por águas pretas - e vale dizer que não foi demonstrado cálcio tanto na água quanto no solo (Williams et al., 1974). Existe uma capacidade de adaptação à baixa ingestão de cálcio no ser humano (Irwin \& Reinholz, 1973; Borle, 1974) .

Não existem dúvidas de que a incorporação adequada de fluor nos dentes, particularmente nas camadas externas do esmalte, aumenta a resistência dos dentes às cáries (Scherp, 1971). O fluor promove a remineralização e o efeito de endurecimento da saliva sobre o esmalte do dente recém-surgido (Wei, 1974). De todas as medidas preventivas disponiveis atualmente, tanto a ingestão de água fluoretada como a suplementação de fluor durante a época do desenvolvimento dental, produzem os melhores efeitos na redução de cáries (Gibbons \& Van Houte, 1975). O consumo de água contendo 1 p.p.m. de fluor reduz a 
incidência de cáries entre $50-60 \%$ nos dentes permanentes (Wei, 1974).

A água potável de Manaus, não contém fluor ainda. Manaus já possui o equipamento necessário para a fluoretação de água instalado (Grinplastch, 1974). A necessidade de importar flurosilicato de sódio ou ácido fluorossilícico a um alto custo, é a razão pela qual o equipamento não foi utilizado. Agora, entretanto, com auxilio do Ministério da Saúde e a Secretaria de Saúde do Estado do Amazonas, a água será logo fluoretada.

Os produtos químicos são caros, mas em comparação com o custo convencional do tratamento dentário, tornam-se compensadores. O fluoreto de cálcio comumente conhecido como fluorita, é largamente encontrado no Brasil e bons resultados estão sendo obtidos com o uso deste produto químico em várias cidades do Brasil (Grinplastch, 1974). É mais barato, e não há necessidade de importar.

Apesar de apenas $57 \%$ das casas em Manaus terem água encanada (Estudo Específico n. $\left.{ }^{41}, 1974\right)$, a fluoretação imediata da água potável asseguraria, pelo menos, a redução da incidência de cáries neste setor da população, a um custo mínimo. Infelizmente, este é o setor da população que mais tem recursos para conseguir tratamento dentário conservativo.

A fortificação de alimentos, tais como farinha ou sal, também já foi tentada em outros lugares, mas produziu dificuldades práticas e econòmicas, comparadas com a fluoretação da água (Wei, 1974). Por cutro lado, em pequenas e isoladas localidades amazônicas, a fluoretação do sal de cozinha pode ser a soluçāo mais prática do que a fluoretação da água, já que foi provado ser aquela tão eficaz e segura quanto esta, em comunidades isoladas semeIhantes na Colômbia (Mejia, et al., 1976). É impossivel toda a Bacia Amazônica possuir água encanada, principalmente na Zona rural, onde moram $60 \%$ (Anuário Estatístico do Amazonas, 1975) da população total, mas o sal de cozinha seria uma solução viável, que atingiria a todos.

Quanto aos fatores dietéticos, seria útil realizar estudos sobre a validade da adição de fasfato a um alimento comumente consumido, mas as poucas tentativas clínicas realizadas com seres humanos não são encorajadoras (Gibbons \& Van Houte, 1975). Por outro lado. um programa de ação promovendo o consumo de açúcar local não refinado, poderia trazer muitos beneficios além de reduzir cáries dentárias.

A aplicação tópica de preparados de fluor é geralmente menos efetiva enquanto dentifrícios contendo fluor, são pouco eficazes (Gibbons \& Van Houte, 1975). Para uma região como a Amazônia, a fluoretaçăo do sal de cozinha pode ser a melhor solução para reduzir a cárie dental em áreas rurais e urbanas, tanto para o ricn quanto para o pobre.

\section{CONCLUSÃo}

Os resultados, apesar de serem de uma amostra pequena, mostram que a incidência de cáries é semelhante à do resto do Brasil e do mundo, além de ser pior do que foi encontrado pelo Inquérito da CNA em áreas urbanas da A:mazônia, vinte anos atrás (Silva, 1959). Os serviços de atendimento dentário são precários e, para a maioria da população, a única solução para o problema é a extração do dente comprometido, quando a dor se torna insuportável. A provisão de um amplo serviço de tratamento dentário seria extremamente cara e impraticável diante da realidade da Bacia Amazônica.

A cárie é uma moléstia infecciosa, e, apesar de ser possível imunizar pessoas contra as bactérias responsáveis (Mickalek et al., 1976). é improvável que aconteça em larga escaia dentro da próxima década.

A prática corrente de introduzir a alimentação artificial nas duas primeiras semanas de vida, além de ser negativa às chances de sobrevivência da maioria das crianças em Manaus (Shrimpton \& Giugliano, 1977) contribui para a rarefação do esmalte e conseqüentemente, aumenta a susceptibilidade dentária aos ataques cariogènicos. Sugere-se que a alimentação artificial seja fortemente combatida em todas as maternidades do Estado do Amazonas. A amamentaçăo deve ser ativamente promovida para a saúde e benefício da mãe amazonense, sua criança e seus dentes. 
Para uma região como a Amazônia, a fluoretação do sal de cozinha pode ser a melhor solução para reduzir a cárie dental em áreas rurais e urbanas, atingindo tanto aos ricos quantos aos pobres.

\section{SUMMARY}

The problem of dental caries in Manaus, Amazonas, is discussed. Results are presented showing $77.7 \%$ incidence in 193 children between one and eight years of age. The factors involved in the evolution of dental caries are described and possible means of atacking the problem considered. The fluoridization of the local water supplies will only reach more priveleged sectors of the population in urban areas. The possibility of using a vehicle such as salt in order to benefit more of the population should be investigated. Promotion of breast feeding at least in the first weeks of life should be stimulated.

\section{BIBLIOGRAFIA CITADA}

\section{ANUÁRio Estatístico do Amazonas}

1975 - Apud: Secretaria do Planejamento e Coordenação Social, Comissão de Desenvolvimento do Estado do Amazonas, $303 \mathrm{p}$

BORLE, A.B.

1974 - Calcium and Phosphate Metabolism. Ann. Rev. Physiol. Comroe Jr., J.H.; Sonnenschein, R.R.; Zierler, K.L. Vol. 36, 361-390. Annual Reviews Inc., Palo Alto, California, $583 \mathrm{p}$.

ESTUDO ESPECÍ́FICO No 41

1974 - Apud: CODEAMA, SEPLAN, Governo do Estado do Amazonas, Manaus.

GEISLER, R. \& SCHNEIDER, J.

1976 - The Element matrix of Amazon waters and its relationship with the mineral content of fishes. Amazonia, Kiel $6(1): 47-65$.

Gibbons, R.J. \& van Houte, J.

1975 - Dental caries in Ann. Rev. Med., Selected Topics in the Clinical Sciences Vol. 26:121-136. $610 \mathrm{p}$.

Gitugliano, R. \& Shrimpton, R.

1977 - Estudo Antropométrico e clínico do estado nutricional em um grupo de crianças pré-escolares de Manaus. Acta Amazonica $7(3): 389-394$. s/d - Estudo Antropométrico e clínico do estado nutricional em crianças no primeiro ano escolar de Manaus. Em prepa ração.

GRINPLASTCH, B.S.

1974 - Fluoretação de águas no Brasil. Boleletin de la Ofícina Sanitária Panamericana $16(4): 321-330$.

HANSON, E.

1933 - Malnutrition in the Amazon Basin. Science 78:33-38.

HYTten, F.E. \& LeITCH, I.

1964 - The physiology of human pregnancy, Oxford, Blackwell Scientific Publications, p. 318-353.

INTERDEPARTMENTAL COMMITTEE ON NUTRITION FOR NATIONAL DEFENSE

1963 - Manual for nutrition surveys. National Institute of Health, Bethesda, M.D. 2nd. Ed., 327 p.

IRVING, J.T.

1957 - Influence of hormones and diet on bone and teeth Harrusm R.S., Marian, G.F., Thiman, K.V., Eds. In: Vitamins and Hormones. Academic Press, N.Y. p. 221-323.

IRWIN, I.M. \& REINHOLZ, E.W.

1973 - Conspectus of research on calcium requirements of man. J. Nutr. 103: 1021-1095.

JAFFe, E.; SNodgrass, G.J. \& Stimmler, A.I.

1973 - Dental defects associated with neonatal symptomatic hypocalcaemia. Arch. Dis. Child., 48:217-220.

Krook, L; Lutwak, L; Whalen, J.P.; Henrikson, P.A.; LESSER, G.V. \& URIS, R.

1972 - Human periodontal disease - morphology and response to calcium therapy. Cornell Vet. 62:32-53.

LAMBrou, D.B.

1974 - A method for the intraoral determination of the cariogenicity of food stuffs: a preliminary report. J. Dent. Res. 53(6):1450-1454.

LIRA, M.B.

1960 - Levantamento de dados alimentares em cidades do interior amazônico. Rev. Bras. Med. 17(7):636.

LOWENSTEIN, F.W.

1967 - Report on nutrition surveys in $11 \mathrm{Bra}$ zilian Amazon communities between 1955-1957. Atas do Simpósio sobre a Biota Amazônica 6 (Patologia). 
MCCLURE, F.J.

1960 - The cariostatic effect in white rats of Phosphorus and Calcium supplements added to the flour of bread formulas and to bread diets. J. Nutr. 72:131-136.

mejia, R.; Espinal, F.; Velez, H. \& Aguirre, M.

1976 - Fluoruración de la sal en cuatra comunidades columbianos VIII, Resultados obtenidos de 1964 a 1972 . Boletin de la Oficina Sanitária Panamericana, 80(3): 205-219.

Michalek, S.M.; MCGHeE, J.R.; Mestecky, J.; ARNOLD, R.R. \& BOZZO, L.

1976 - Fluoruración de la sal en cuatra comuinducer secretory immunoglobulin A and caries immunity. Science 192: 1238-1240.

SHERP, H.W.

1971 - Dental caries: prospects ior prevention. Science, 173:1199-1205.

SHRIMPton, R. \& Rodrigues, N. De Marco

s/d - Padrões alimentares em Manaus, baseados em dados do Inquérito de Orçamen. tos Familiares da CODEAMA, 1973-74. (em preparação),

SHRIMPtON, R. \& GiUgliano, R.

1977 - Nutrição em lactentes de um bairro de Manaus, Amazonas. Acta Amazonica $7(2): 247-253$.

SILVA, W.

1859 - Inquérito sobre consumo de alimentos e nutrientes, avaliação do estado nutri- tivo e situação econômica da população amazônica. Boletim da Comissão Nacional de Alimentação, 4(2): Julho-setembro, Rio de Janeiro.

Sutmoller, P.; Vahia de Abreu, A.; VAnder Grift, J. \& Sombroek, W.G.

1966 - Mineral imbalances in cattle in the Amazon Valley Department of Agricultural Research, Royal Tropical Institute, Amsterdam. Communication 53: 1-135 p.

THOMSON, A.M. \& BLACK, A.E.

1975 - Nutritional aspects of lactation. Bull. World Health Organiz, 52:163-177.

Velez Boza, F. \& Baumgartner, J.

1962 - Estudio general, clínico y nutricional en tribus indígenas del Território $\mathrm{Fe}$ deral Amazonas de Venezuela. Arch. Venezol. Nutrición 12:143-225.

WEI, H.Y.S.

1974 - Nutritional aspects of dental caries, in Fomon, S.L. ed. Infant Nutrition. W.B. Saunders Co. Philadelphia, 575 p.

Whliams, W.A.; LoOMis, R.S. \& Alvim, P.T.D.

1974 - Environments of evergreen rain forests on the Lower Rio Negro, Brazil. Tropical ecology 13(1):65-78.

WILSON, E.D.; FISHER, K.H. \& FUQUA, M.E.

1975 - Principles of Nutrition. John Willey \& Sons, 598 p. 